\title{
Dinámica y distribución de la productividad del trabajo en México después de la apertura comercial
}

\section{Dynamics and distribution of labor productivity in Mexico after trade openness}

\author{
Luis GutiérRez-Flores* \\ Maritza Areli Velázquez-Villalpando** \\ Gustavo FÉLIX-VerduZCo***
}

\begin{abstract}
This document analyzes the dynamic of productivity distribution in Mexico's federal states for the 1994-2009 period. The main objective of the paper is to inquire whether the dynamic process of productivity distribution in the Mexican manufacturing sector has a similar pattern to the one found when discussing income convergence. For such an end, a different methodological tool is used; this tool allows considering the dynamic processes in the productivity levels of the Mexican states. Our results indicate a trend toward a concentration of the manufacturing activity in the country and also toward a separation in productivity levels in recent years.
\end{abstract}

Keywords: productivity, manufacturing industries, Markov chains, regions, polarization, separation.

\section{Resumen}

El presente documento analiza la dinámica en la distribución de la productividad en las entidades federativas de México para el periodo 1994-2009. El objetivo central de la investigación es conocer si el proceso dinámico de distribución de la productividad del sector manufacturero, con el uso de las cadenas de Markov, tiene un patrón similar o no al encontrado cuando se discute acerca de la convergencia en ingresos. Los resultados del trabajo indican una tendencia no únicamente hacia la concentración de la actividad manufacturera del país, sino también hacia una separación en los niveles de productividad en los años recientes.

Palabras clave: Productividad, industria manufacturera, cadenas de Markov, regiones, polarización, separación.

\footnotetext{
* Centro de Investigaciones Socioeconómicas de la Universidad Autónoma de Coahuila. e-mail: luis.gutierrez@uadec.edu.mx

** Doctora en Economía Regional por la Universidad Autónoma de Coahuila. e-mail: maritzaareli@ hotmail.com

*** Centro de Investigaciones Socioeconómicas de la Universidad Autónoma de Coahuila. e-mail: gustavo.felix@uadec.edu.mx
} 


\section{Introducción}

A raíz de la apertura comercial que experimentó la economía mexicana, y más específicamente con la implementación del Tratado de Libre Comercio de América del Norte (TLCAN), el debate acerca de las disparidades regionales se ha acrecentado. No obstante, cabe reconocer que hacia eso apuntan en su mayoría las principales conclusiones de este proceso económico, es decir, las regiones en nuestro país tienden hacia su divergencia y polarización y contradicen así, por ejemplo, a la teoría de la convergencia, misma que sólo ha sido probada en países desarrollados.

Para el caso de México, han sido varios los análisis enfocados a este tema, éstos consideran principalmente el ingreso per cápita antes y después de la apertura comercial, con lo que se ha tenido evidencia de divergencia y polarización interna, lo cual resulta en una segmentación del crecimiento del país en dos grandes regiones muy distantes entre sí.

La productividad del trabajo es una variable que ha sido justificada técnicamente como determinante de los procesos de convergencia-divergencia, pero a pesar de tal afirmación los análisis de las disparidades y de convergencia en México no han sido del todo abordados desde esta perspectiva analítica, es decir, se consideran otras metodologías no paramétricas, tales como las cadenas de Markov.

Una de las aportaciones más relevantes de este documento es la propuesta de una metodología alternativa para evaluar fenómenos como la convergencia regional, la cual se ha caracterizado por utilizar en su mayoría técnicas econométricas; las investigaciones en México que utilizan estas técnicas no paramétricas son relativamente escasas, lo que invita a comparar resultados desde este cuerpo metodológico.

En el presente artículo se tiene por objetivo analizar la distribución y la dinámica en la transición de la productividad del trabajo en el sector manufacturero durante el período posterior a la firma del TLCAN, con lo que se pretende contribuir y fundamentar la teoría de la convergencia-divergencia regional, en términos del desempeño productivo manufacturero.

El marco de referencia que sustenta este análisis evalúa si, después de la firma de dicho acuerdo comercial, las entidades en México han tendido hacia la convergencia productiva regional o si se ha presentado un proceso en el sentido contrario; por lo tanto, la hipótesis a comprobar es que, después de la apertura comercial, la dinámica distribucional de la productividad del trabajo en la manufactura tiene un comportamiento polarizado y persistente entre las regiones.

También es importante identificar la diferencia o similitud en cuanto a resultados desde esta perspectiva metodológica: las cadenas de Markov, 
y verificar si coincide con los resultados divergentes observados a través de estudios econométricos, o bien, si es posible encontrar algún indicio de convergencia regional en México después de la firma del tLCAN.

La estructura del documento se divide en cuatro apartados: en el primero se discuten brevemente algunos trabajos empíricos acerca de la convergencia regional en México a la luz de la apertura comercial; en el segundo se desarrolla y explica la metodología utilizada, en este caso, las cadenas de Markov; en la tercera sección se describe concretamente la distribución de la industria manufacturera y su desempeño productivo para 1993, 1998, 2003 y 2008; la cuarta parte se dedica a la aplicación empírica de la metodología y el análisis de la dinámica de transición de la productividad del trabajo manufacturero, asimismo se evalúa el proceso de convergencia productiva en México, en esta sección también se dedica un apartado especial para el análisis de la maquiladora. Finalmente se presentan las conclusiones.

\section{Apertura comercial y convergencia regional}

La expresión más importante del proceso general de apertura comercial en México ha sido la firma del tLCAN (Rodríguez-Arana, 2009). Sin embargo, a raíz de la firma de éste se han observado crecientes divergencias entre las entidades federativas del país, principalmente en términos del ingreso per cápita (Aroca et al., 2003).

Arriaga-Navarrete et al. (2005) mencionan que el crecimiento económico de México está determinado de manera importante por el grado de especialización de las regiones. En un contexto de apertura comercial, financiera y productiva, la economía mexicana muestra una heterogénea distribución del producto estatal per cápita, diferencia que a su vez se manifiesta en una desigualdad regional en diversos indicadores socioeconómicos.

Ocegueda-Hernández (2007) documenta estadísticamente un proceso de polarización después de la apertura comercial, compara los distintos niveles de crecimiento en los ingresos per cápita de los estados más pobres y más ricos. De 1994 a 2004 la tasa de crecimiento del sur (Michoacán, Guerrero, Oaxaca y Chiapas) fue de 0.4, y para el norte (Baja California, Sonora, Chihuahua, Coahuila y Nuevo León) fue de 1.7 por ciento. Esto ha generado una redistribución del ingreso muy distinta a la esperada, ampliándose la brecha y aumentando la polarización al interior del país, con lo que la idea de la convergencia interregional queda alejada de la realidad.

Ocegueda-Hernández (2007) atribuye las divergencias interregionales a los rendimientos crecientes a escala y a las dinámicas acumulativas 
circulares como mecanismos acrecentadores de las disparidades iniciales. En la descripción de este proceso, varios han sido los factores que han incidido en la desigual evolución de los niveles de ingreso por habitante y su distribución espacial, con los cuales se han marcado las tendencias hacia la polarización regional.

La relación entre apertura y crecimiento es un problema empírico en el cual un eslabón importante es la productividad (Fragoso-Pastrana, 2003), al respecto Esquivel y Messmacher (2002), analizan las fuentes del crecimiento en México de 1960 al 2000, ellos encuentran que el comportamiento de la productividad del trabajo es el principal factor que influye en el patrón de divergencia regional, acentuada por la firma del tLCAN, lo cual origina que los beneficios se hayan distribuido de manera inequitativamente entre regiones y sectores.

Por su parte, Calderón y Tykhonenko (2006) evalúan el proceso de convergencia regional en México, se basan en un análisis econométrico de datos de panel; las principales conclusiones de su investigación son que antes de la apertura comercial (la firma del GATT) existía un proceso de convergencia en el ingreso per cápita entre los estados mexicanos, pero que después de la apertura, y más aún con la firma del TLCAN, las entidades presentaron una divergencia real en su ingreso.

Reconocen, asimismo, que las entidades de la frontera norte fueron las que presentaron un aumento importante del PIB per cápita debido a la mejora en capital humano y a la productividad del trabajo; de acuerdo con estos autores, después de la firma del TLCAN se observó una divergencia interregional con cierta tendencia hacia la polarización, principalmente respecto a las entidades fronterizas del norte. Ponce de León (2012) coincide con estos resultados sobre divergencia en las regiones de México después de la firma del TLCAN.

Como ha sido discutido por varios autores, con la apertura comercial, y más específicamente con el TLCAN, el proceso divergente interregional en México tiene tendencias del crecimiento que se perfilan más hacia una polarización que a una convergencia interregional. Autores como Esquivel y Messmacher (2002) mencionan que el factor detonante del proceso divergente en México ha sido la productividad del trabajo. Sin embargo existen pocas evidencias concretas acerca de la convergencia interregional que consideran como variable de análisis a la productividad; destacan los trabajos de Alañón (1999), Dall'erba (2005), Valdivia-López (2007) y De León-Arias (2008),

Por otro lado, también es muy importante el aspecto metodológico utilizado, la mayoría de la literatura que aborda el tema de la convergencia regional aplica técnicas de análisis paramétrico y toma como referencia el ingreso per cápita. En la literatura económica, autores como 
Barro et al. (1991) han realizado estudios dedicados al análisis de convergencia, quienes dentro del marco neoclásico del crecimiento, aseguran que se puede llegar a una convergencia entre países o regiones a una tasa de $2 \%$ anual.

Sin embargo, Durlauf y Quah (1998) realizan una amplia crítica a tales postulados, demostrando que dadas las condiciones iniciales de las regiones o países, es más probable que se mantenga esa tendencia divergente o peor aún que se acentúen las disparidades. Los autores demuestran cómo es que por la propia naturaleza de los métodos paramétricos, independientemente de la tendencia del crecimiento, se puede incurrir en sesgos de estimación y llegar a la tasa de convergencia del $2 \%$ para la gran mayoría de regiones o países analizados.

Por lo tanto, dado el debate entre las posturas teóricas y técnicas, puede afirmarse que hay evidencia suficiente sobre los análisis de convergencia pero resulta interesante analizarla utilizando como variable la productividad del trabajo, bajo una perspectiva como la planteada por Quah (1993, 1996), ${ }^{1}$ Durlauf y Quah (1998), así como comparar resultados entre las alternativas metodológicas.

Al utilizar esta metodología alternativa, a nivel internacional también se ha encontrado evidencia del fenómeno de divergencia regional. El trabajo de Arrufat et al. (2005) analiza la movilidad regional en Argentina aplicando las cadenas de Markov, las cuales confirman los resultados divergentes en concordancia con los argumentos de Quah (1993).

Por su parte, Cáceres-Apolinario et al. (2009) utilizan también esta metodología, realizan un comparativo donde evalúan el proceso de convergencia entre 22 países en vías desarrollo, sus resultados apuntan a encontrar cierta convergencia sólo entre nueve países: Chile, México, China, Malasia, Taiwán, Tailandia, Israel, República Checa y Marruecos; identifican en ese estudio cierta convergencia de clubes a nivel internacional, sin embargo resulta interesante evaluar este proceso al interior de cada país, tal como se realiza en el presente documento.

Un aspecto relevante en este contexto es el hecho de considerar que únicamente el trabajo de Quilis (1997) está circunscrito a esta delimitación metodológica, es decir, que se analiza la convergencia interregional en Espańa considerando la productividad del trabajo con un análisis no paramétrico, se utilizan cadenas de Markov, lo cual invita a realizar un estudio similar para el caso mexicano.

\footnotetext{
${ }^{1}$ Quah (1996) menciona que debido a la metodología utilizada para medir la convergencia se incurre en ciertos sesgos al predecir la tasa de convergencia del $2 \%$ y hace referencia a las técnicas econométricas, sin embargo, reconoce que es más probable encontrar convergencia de clubes o una polarización en el crecimiento económico, idea que se aleja del concepto general de convergencia.
} 


\section{Método: las cadenas de Markov}

Las cadenas de Markov se describen como un proceso estocástico utilizado frecuentemente desde la ciencia económica para describir la movilidad de los agentes económicos entre distintos estados (García-Verdú, 2005). Se trata de cierto tipo de procesos que "carecen de memoria", es decir, que la transición de un estado sólo depende del estado presente en el que se encuentra el sistema (Bedoya y Barrera, 2006). En general, la interpretación de las cadenas de Markov tiene como objetivo estimar la dinámica de alguna distribución, en este caso, de la productividad del trabajo en función de las probabilidades de transitar del estado $i$ al $j$ y del número de iteraciones que se realicen (Gutiérrez-Flores et al., 2011).

El modelo de Markov utilizado en este documento se basa en el trabajo pionero de Quah (1993) y es retomado por autores como Quilis (1997), García-Verdú (2005), Cáceres-Apolinario et al. (2009), GutiérrezFlores et al. (2011), entre otros. La formalización del modelo siguiendo a Quah (1993) es la siguiente:

La variable en estudio (Productividad del trabajo) para cada entidad en el periodo $t$ se representa por $(\mathrm{P} t)$. La secuencia de observaciones en el tiempo de la productividad $(\mathrm{P} t)$ es un proceso estocástico con un parámetro de tiempo discreto. A la primera observación se le llama estado inicial y a los subsecuentes estados $t=2,3, \ldots . n$, la observación $(\mathrm{P} t)$ se denomina Estado del proceso en el tiempo $t$.

De acuerdo con lo anterior, en cada periodo $t$ existen $n$ estados mutuamente excluyentes, en los cuales cada región tiene que ocupar uno de esos estados. El modelo para la productividad del trabajo está dado por un vector de probabilidad inicial $\left(\pi_{1}\right)$, mismo que describe la probabilidad de los posibles valores del estado inicial $P_{1}$ :

$$
\pi_{1 i}=\operatorname{Pr}\left(P_{1}=p_{1}\right), \quad \pi_{1 i} \geq 0 \text { para } i=1, \ldots \ldots . ., \sum_{t=1}^{n} \pi_{1 i}=1
$$

Para cada estado subsecuente $\mathrm{P}_{t+1}, t=1,2,3, \ldots .$. , cada probabilidad condicional se presenta de la siguiente forma:

$$
\operatorname{Pr}\left(P_{t+1}=\left(p_{t+1} \mid P_{t}=p_{t}, P_{t-1}=p_{t-1}, \ldots, P_{1}=p_{1}\right)\right.
$$

Una cadena de Markov es un tipo de proceso estocástico determinado para cualquier tiempo $(t=1,2,3 \ldots)$ y para cualquier secuencia de estados posible $\left(p_{1}, p_{2}, \ldots \ldots t\right)$ : 


$$
\operatorname{Pr}\left(P_{t+1}=p_{t+1} \mid P_{t}=p_{t}, P_{t-1}\right)=p_{t-1}, \ldots \ldots, P_{1}=p_{1}
$$

Esto quiere decir que la probabilidad de todos los estados futuros $\mathrm{P}_{\mathrm{t}+\mathrm{k}}, \mathrm{k} \geq 1$, depende solamente del estado $\mathrm{P}_{\mathrm{t}} \mathrm{y}$ no de los estados previos (Bedoya y Barrera, 2006). A la probabilidad condicional $\operatorname{Pr}\left(P_{t+1}=p_{t+1} \mid P_{t}=p_{t}\right)$ se le conoce como probabilidad de transición. Tales probabilidades tienen el mismo valor en cada tiempo, de modo que la cadena de Markov tiene las siguientes probabilidades de transición.

$$
\operatorname{Pr}\left(P_{t+1}=p_{j} \mid P_{t}=p_{i}\right)=p_{i j}
$$

Donde $p_{i j} \geq 0$ es la probabilidad que una economía (en este caso una entidad) estará en el estado $j$ en el siguiente periodo, dado el estado $i$. Las probabilidades del movimiento de valor de un estado a otro en un periodo se establecen utilizando una matriz cuadrada ( $n x$ n) de transición $P$ :

$$
\dot{P}=\left(\begin{array}{ccc}
p 11 & \cdots & p 1 n \\
\vdots & \ddots & \vdots \\
p 1 n & \cdots & p n n
\end{array}\right)
$$

Cada fila de la matriz anterior debe satisfacer la siguiente condición:

$$
\sum_{j=1}^{n} p_{i j}=1 \text { para } i=1, \ldots n
$$

Si la cadena de Markov tiene una transición de probabilidades estacionaria, dada una transición de la matriz $P$ en una sola etapa, se pueden calcular las probabilidades de movimiento de cualquier valor del estado, a cualquier otro valor de otro estado en dos periodos como $P^{2}$, desde la siguiente expresión:

$$
\begin{aligned}
& \operatorname{Pr}\left(P_{t+2}=y_{j} \mid P_{t}=p_{i}\right)=\sum_{h=1}^{n} \operatorname{Pr}\left(P_{t+1}=p_{j} \mid P_{t}=p_{h}\right) \operatorname{Pr}\left(P_{t+1}=p_{h} \mid P_{t}=p_{i}\right) \\
& =\sum_{h+1}^{n} p_{i h} p_{h j}=p_{i j}^{2}
\end{aligned}
$$


Similarmente, se pueden calcular las probabilidades del movimiento de cualquier valor del estado inicial a otro valor del estado en $k$ periodos como $P^{k}$ :

$$
\operatorname{Pr}\left(P_{t+k}=p_{j} \mid P_{t}=y_{i}\right)=p_{i j}^{k}
$$

La probabilidad de distribución incondicional de $\mathrm{P}_{\mathrm{t}}$ está dada por:

$$
\begin{aligned}
& \pi_{2}=\operatorname{Pr}\left(P_{2}\right)=\pi_{1} P \\
& \pi_{3}=\operatorname{Pr}\left(P_{3}\right)=\pi_{1} P^{2}=\pi_{2} P \\
& \vdots \\
& \pi_{k}=\operatorname{Pr}\left(P_{k}\right)=\pi_{1} P^{k}=\pi_{k-1} P
\end{aligned}
$$

Para las ecuaciones precedentes se puede decir que la probabilidad de distribución incondicional evoluciona de acuerdo a lo siguiente:

$$
\pi_{t+1}=\pi_{t} P
$$

Una distribución es llamada invariante o ergódica si cumple lo siguiente:

$$
\pi_{t+1}=\pi_{t}=\pi^{*} \text { para todo } t
$$

Esto es, que la distribución incondicional permanece constante en el tiempo. De esta forma, una distribución ergódica debe satisfacer la expresión:

$$
\begin{aligned}
& \pi^{*}=\pi^{*} P \\
& \pi^{*}(I-P)=0
\end{aligned}
$$

Transponiendo esta última ecuación se tiene:

$$
\left(I-P^{\prime}\right) \pi^{* \prime}=0
$$

Lo cual determina $\pi^{*}$ como un eigenvector ${ }^{2}$ asociado con una unidad de eigenvalor de $P^{\prime}$. El hecho de que $P$ sea una matriz estocástica garantiza que tiene por lo menos un eigenvalor unitario y, que hay algún $\pi^{*}$

\footnotetext{
${ }^{2}$ Eigenvector: por definición es un vector característico o vector propio de una matriz A.
} 
que satisface la ecuación (13). Dependiendo de $P$, una distribución ergódica puede o no puede ser única. En particular, si cada entrada de la matriz $\mathrm{P}$ es estrictamente positiva, luego entonces existe una única distribución ergódica llamada distribución estacionaria.

\section{Productividad en la industria manufacturera mexicana: su distribución y dinámica de transición}

La configuración espacial de la industria manufacturera mexicana se basa en dos esquemas que guían su funcionamiento. Fragoso-Pastrana (2003), Trejo-Nieto (2010) y Velázquez (2010), entre otros, argumentan que la industria maquiladora se articula y debe su desempeńo al mercado internacional, mientras que la industria no maquiladora se integra a la dinámica económica nacional.

Al considerar la localización industrial en México, se identifica que iniciada la apertura comercial, existe cierta concentración geográfica de la industria y una tendencia a la aglomeración en los estados del norte del país, ${ }^{3}$ es decir, que existe una clara relación entre estados con mayor participación en los sectores exportadores y aquellos con niveles de productividad por encima de la media. Es por ello que a partir de dicha integración, la reubicación territorial de la industria nacional y la concentración de los sectores es observada en ciertas áreas geográficas; es este hecho un indicio de diferencias regionales en su capacidad de atracción de la inversión asociada a economías externas (Velázquez, 2010).

\subsection{Descripción de datos}

Para el análisis de la industria manufacturera, los datos fueron tomados de los censos económicos 1994, 1999, 2004 y 2009. Específicamente el Valor agregado y el Personal ocupado. La primera de estas variables, al ser un dato monetario, se tuvo que deflactar con el deflactor implícito del рів para las actividades manufactureras por entidad para 1993, 1998 y 2003 a precios del 2008.

Para realizar el análisis del caso maquilador, la información se obtuvo de una base de datos que proporciona el INEGI y se llama Industria Maquiladora de Exportación (IME), la cual sólo considera algunas entidades del país: Aguascalientes, Baja California, Baja California Sur, Chihuahua, Coahuila, Distrito Federal, Durango, Guanajuato, Jalisco, México, Nuevo León, Puebla, San Luis Potosí, Sinaloa, Sonora, Tamaulipas, Yucatán y Zacatecas.

\footnotetext{
${ }^{3}$ Una descripción más amplia de este proceso se puede ver en Dávila-Flores (2005).
} 
La IME reporta datos mensuales de 1996 al 2006, por lo que se obtuvo un promedio anual para 1996, 2000 y 2006 con el fin de evaluar las cadenas de Markov.

Los análisis de productividad del trabajo se pueden obtener con diversas metodologías, una de las más utilizadas es aquélla que se deriva de la razón del Valor agregado sobre el Personal ocupado (Sobrino, 1999; Fragoso-Pastrana, 2003 y Díaz-Bautista, 2008), para efectos de un análisis comparativo entre regiones manufactureras, ${ }^{4}$ enseguida se presenta el indicador de productividad por entidad con datos de los censos económicos antes mencionados, que a su vez es obtenido mediante lo siguiente:

$$
P T=\frac{V A}{P O}
$$

Donde: $P T=$ Productividad del Trabajo

$$
V A=\text { Valor Agregado Censal Bruto } 5
$$

$P O=$ Personal Ocupado en la Industria Manufacturera

Con el coeficiente anterior fue posible identificar las diferencias que se tienen en la productividad en las distintas entidades en los tres periodos de análisis, cuyas tendencias se presentan en los siguientes mapas.

Los mapas anteriores reflejan algunas situaciones interesantes; en primer lugar la industria manufacturera se distribuye desigualmente entre las entidades del país (Velázquez, 2010), incluso, su desempeño productivo es mucho más heterogéneo y con notorios cambios entre cada año de análisis.

Los mapas reflejan en el color más claro bajos niveles de productividad, mientras que la mayor productividad se muestra en el tono más oscuro. En breve resumen, para 1993, las entidades más productivas resultaron ser Distrito Federal, Estado de México, Jalisco y Nuevo León, mientras que los menos productivos fueron Guerrero, Campeche, Chiapas, Yucatán y Zacatecas. En 1998 hubo algunos cambios en los niveles de productividad en las entidades, pero en general se mantuvo la misma tendencia en cuanto a los más y los menos productivos; para el 2003, el patrón cambió drásticamente, ya que entidades como Nuevo León

\footnotetext{
${ }^{4}$ El INEGi publica la Encuesta Industrial Mensual (EIM) considerando únicamente a aquellas entidades que tienen una presencia significativa de la industria manufacturera.

${ }^{5}$ El Valor Agregado Censal fue deflactado con el deflactor implícito del pIB para las actividades manufactureras por entidad federativa para 1993, 1998 y 2003 a precios del 2008, lo que lo hace comparable entre años.
} 


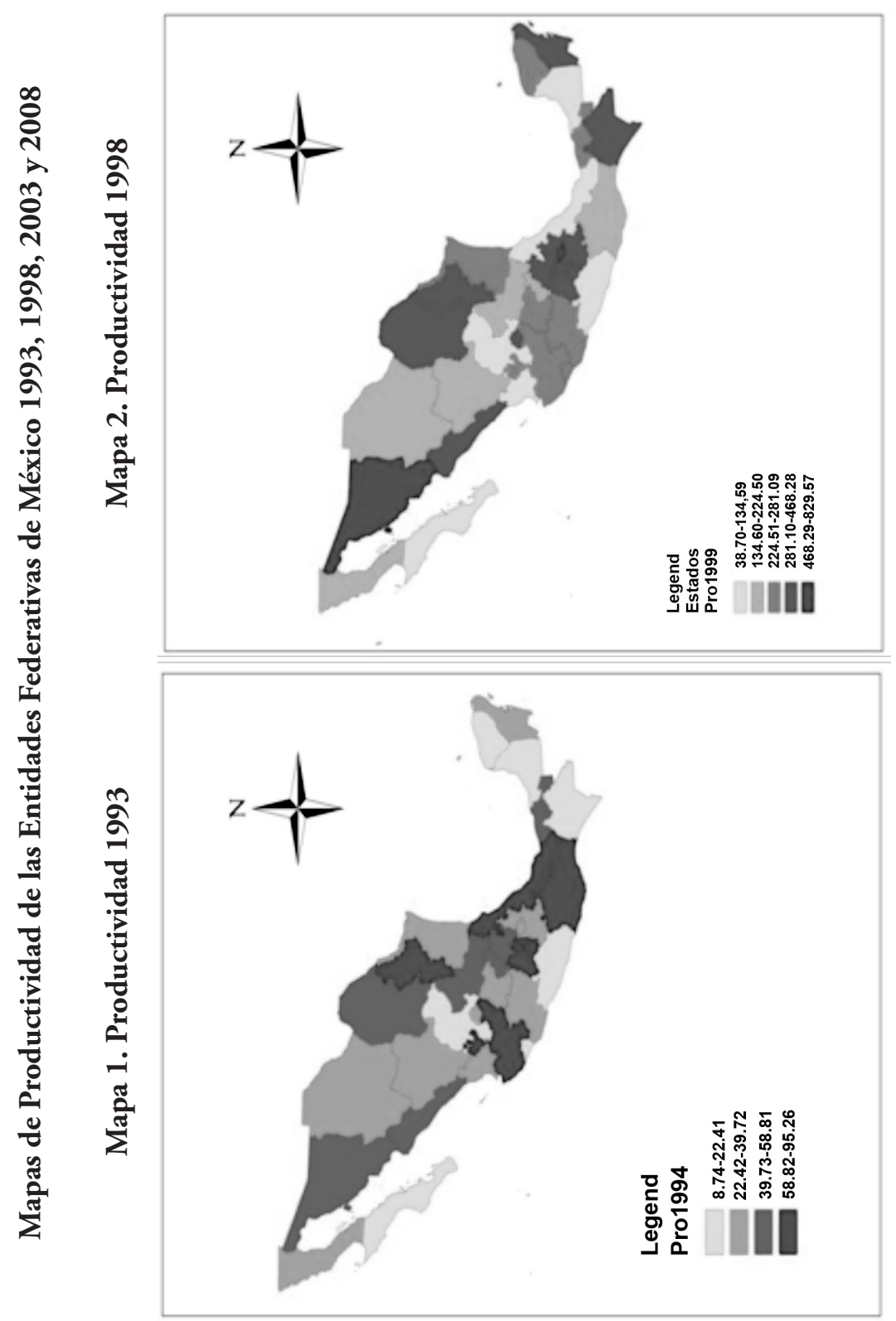



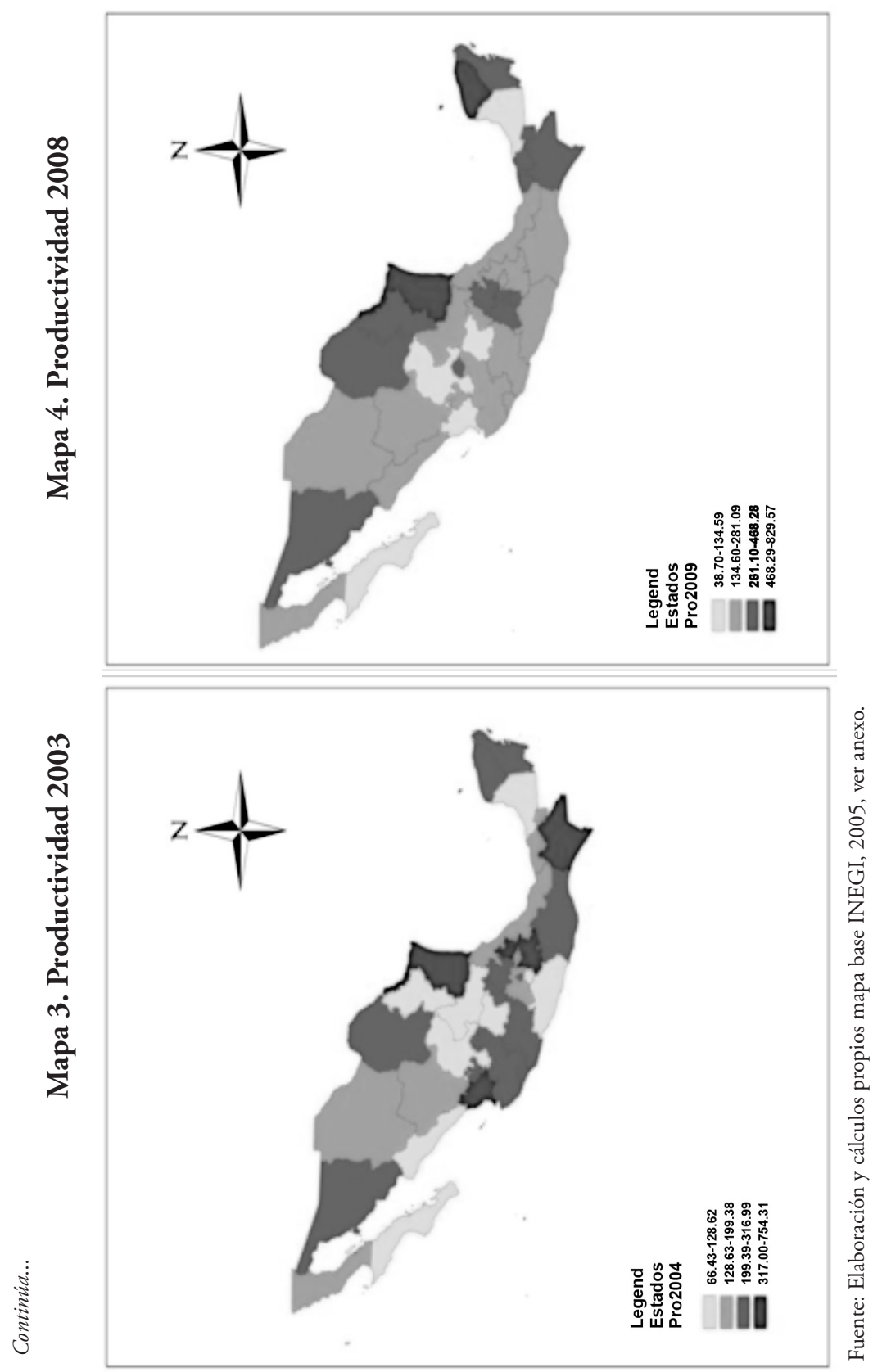
y San Luis Potosí arrojaron índices en el rango más bajo en cuanto a su productividad, mientras que Chiapas se ubicó dentro de los más productivos. ${ }^{6}$

Para el último año de registro, Aguascalientes y Yucatán fueron los más productivos; el Estado de México, Nuevo León y Sonora, entre otros, se mantuvieron en un rango medio. La apreciación general del último mapa nos podría dar un indicio de convergencia productiva (a la baja), pues la mayoría de las entidades se sitúan en rangos de productividad medios, lo que disminuye considerablemente los altos contrastes en el mapa.

\section{Dinámica de distribución de la productividad del trabajo en México}

La sección anterior muestra claramente las diferencias en cuanto a la distribución de la manufactura y sus niveles de productividad, sin embargo, es necesario analizar cómo ha sido esa dinámica de transición entre las entidades de un año a otro, se considera para ello el cálculo de la productividad en función de los datos proporcionados por los censos industriales para los años de 1993, 1998, 2003 y 2008.

Con lo anterior se pretende dar cuenta de la dinámica de transición desde la firma del TLCAN, para así poder evaluar si la productividad del trabajo que tiende hacia la convergencia como lo demuestra Quilis (1997) para el caso de España; el procedimiento es el siguiente:

1) Primero es necesario jerarquizar de menor a mayor los niveles de productividad para el primer año de análisis y así contrastarlo con el segundo año, es decir, analizar la dinámica distribucional de la productividad en su transición de 1993 a 1998.

2) Posteriormente se elaboran intervalos de los indicadores de productividad de manera arbitraria. ${ }^{7}$ De acuerdo con Quah (1993), la mayoría de estas aplicaciones dividen la muestra en quintiles, mismos que pueden incluso ser de diferente tamaño.

3) Se realiza un comparativo de las entidades en función del intervalo al que pertenecían en el año inicial con el intervalo al cual se trasladaron en el siguiente año o estado (en su caso).

4) Con lo anterior, se obtiene la matriz de probabilidades de transición en función del número de entidades que hayan permanecido en

\footnotetext{
${ }^{6}$ Esta situación valdría la pena explorarla con otro indicador de productividad, ya que como comentan varios autores, la relación entre Valor agregado y Personal ocupado puede arrojar sesgos de estimación debido al poco Personal ocupado en alguna de las entidades.

${ }^{7}$ En este trabajo, los rangos utilizados son cinco, en relación con los indicadores de productividad de las entidades.
} 
el mismo intervalo de productividad y de las que se movieron a algún otro.

5) La sumatoria de las filas de la matriz de transición tiene que ser igual a la unidad.

6) El vector de estado inicial se obtiene dividiendo el número de entidades en cada intervalo en el período de inicio entre el total de las mismas.

Al considerar lo anterior se presentan las matrices de transición que muestran la dinámica de la productividad en los años observados:

El vector de estado inicial queda conformado como sigue:

$$
\pi_{1}=\left[\begin{array}{lllll}
0.19 & 0.19 & 0.19 & 0.22 & 0.22
\end{array}\right]
$$

Se interpreta como que existe $19 \%$ que se mantiene en los primeros tres intervalos y $22 \%$ en los dos últimos rangos de productividad, mismos que reflejan una mejoría en el rendimiento productivo para las entidades, o equivalentemente, que existe una mayor probabilidad de mejorar la productividad para cada entidad.

La primera transición identificada de la productividad del trabajo de 1993 a 1998 se refleja en la matriz de probabilidades anterior (tabla 1). Destaca el hecho de que evidentemente se presentaron cambios en cuanto los rangos de productividad, situación que se aprecia al haber proporciones de participación en cada intervalo; de no ser así tendría que haber el valor de uno en la diagonal principal.

\section{Tabla 1}

\section{Matriz de Transición 1993-1998}

\begin{tabular}{ccrrrrrr}
\hline $\begin{array}{c}\text { Num. } \\
\text { Entidades }\end{array}$ & Intervalos & 1 & 2 & 3 & 4 & 5 & Suma \\
\hline 6 & 1 & 0.67 & 0.33 & 0 & 0 & 0 & 1 \\
6 & 2 & 0.17 & 0.5 & 0.17 & 0 & 0.17 & 1 \\
6 & 3 & 0 & 0.17 & 0.5 & 0.17 & 0.17 & 1 \\
7 & 4 & 0.14 & 0 & 0.29 & 0.29 & 0.29 & 1 \\
7 & 5 & 0 & 0 & 0 & 0.57 & 0.43 & 1 \\
\hline
\end{tabular}

Fuente: elaboración y cálculos propios con base en datos de INEGI (1994, 1999, 2004, 2005 y 2009). 
La diagonal principal, al tener valores decimales mayores de cero, refleja el cambio o transición de entidades de un intervalo a otro, no obstante, al ser este valor (diagonal) mayor que cualquier otro elemento de las filas correspondientes, nos indica que existe una mayor probabilidad de permanecer en ese mismo intervalo que cambiar de un estado a cualquier otro; a excepción del último rango, donde se refleja una mayor probabilidad de moverse hacia un intervalo de menor valor en cuanto a productividad, que mantenerse en el rango con los más altos niveles de tal indicador.

Sin embargo, para las regiones que se encontraron inicialmente en el rango de menor productividad, la matriz muestra que no hubo probabilidades de trasladarse hacia el rango de mayor productividad. Las entidades que permanecieron de 1993 a 1998 en el mismo nivel productivo son, para el primer rango: Baja California, Campeche, Chiapas y Guerrero; mientras que las entidades que mejoraron su nivel en este periodo fueron Colima y Zacatecas.

Para el segundo rango, las entidades que se mantuvieron fueron: Baja California, Chihuahua y Durango, mientras que únicamente Nayarit mejoró su ubicación, en cambio Yucatán y Quintana Roo empeoraron la posición. Para el tercer rango las entidades que se mantuvieron fueron Michoacán, Puebla y Tlaxcala, las que mejoraron fueron Aguascalientes y Guanajuato, mientras que Tamaulipas empeoró su rango de rendimiento.

Para el cuarto rango, las entidades que se mantuvieron fueron Hidalgo y Sonora; Coahuila, San Luis Potosí, Querétaro y Tabasco tuvieron mejoría, mientras que la única entidad que empeoró fue Sinaloa. Para el último rango las entidades que permanecieron en el mayor intervalo de productividad fueron México, Morelos y Veracruz; las que empeoraron Distrito Federal, Jalisco, Nuevo León y Oaxaca.

El estado de transición de 1998 a 2003 se muestra en la tabla 2:

Tabla 2

Matriz de Transición 1998-2003

\begin{tabular}{ccrrrrrr}
\hline $\begin{array}{c}\text { Núm. } \\
\text { Entidades }\end{array}$ & Intervalos & \multicolumn{1}{c}{1} & 2 & 3 & 4 & 5 & Suma \\
\hline 6 & 1 & 0.5 & 0 & 0 & 0.17 & 0.33 & 1 \\
6 & 2 & 0.17 & 0.33 & 0.17 & 0.17 & 0.17 & 1 \\
6 & 3 & 0 & 0.33 & 0.17 & 0.17 & 0.33 & 1 \\
7 & 4 & 0 & 0.29 & 0 & 0.43 & 0.29 & 1 \\
7 & 5 & 0.14 & 0.14 & 0.57 & 0.14 & 0 & 1 \\
\hline
\end{tabular}

Fuente: elaboración y cálculos propios con base en datos de INEGI (1994, 1999, 2004, 2005 y 2009). 
Similarmente, al evaluar la dinámica de transición de distribución de la productividad de 1998 a 2003, se observa que la posición en la diagonal principal sigue reflejando esas variaciones que muestran una mayor persistencia en algunos de ellos, como es el caso del primer rango, el cual quiere decir que existe $50 \%$ de probabilidades de cambiar de un rango a otro, así como la misma proporción de quedarse en el mismo nivel de productividad; el último rango refleja que una mayor productividad no se mantuvo, es decir que no hay probabilidades, al menos en este periodo, de que las entidades que reportaron los más altos índices de productividad se hayan quedado en la clasificación de las entidades más productivas. Sin embargo las entidades que iniciaron en el menor rango de productividad tienen $33 \%$ de posibilidades de trasladarse hacia al rango de mayor nivel productivo.

De acuerdo con la transición de las entidades en estos años, para el primer intervalo los estados que se mantuvieron fueron Baja California Sur, Campeche y Guerrero, mientras que las entidades que transitaron a un mayor rango productivo o mejoraron fueron Chiapas, Quintana Roo y Yucatán. Para el segundo rango las entidades que permanecieron fueron Baja California y Durango, las que mejoraron: Chihuahua, Oaxaca y Tamaulipas; Zacatecas empeoró.

Para el tercer rango, únicamente el Distrito Federal se mantuvo en el mismo intervalo, por su parte Michoacán, Puebla y Nayarit mejoraron su posición; Tlaxcala y Sinaloa la empeoraron.

En cuanto a la transición de las entidades correspondientes al cuarto rango se observa que Aguascalientes, Hidalgo y Sonora se mantuvieron, mientras que Colima y Jalisco mejoraron su posición; por su parte, Guanajuato y Nuevo León la empeoraron. Para el quinto intervalo se tiene que todas las entidades que se ubicaban en este nivel en 1998, empeoraron para el 2003, son Coahuila, México, Morelos, San Luis Potosí, Tabasco, Querétaro y Veracruz.

Las probabilidades de transición de 2003 a 2008 se muestran en la tabla 3 .

Tabla 3

Matriz de Transición 2003-2008

\begin{tabular}{ccrrrrrr}
\hline $\begin{array}{c}\text { Num. } \\
\text { Entidades }\end{array}$ & Intervalos & 1 & 2 & 3 & 4 & 5 & Suma \\
\hline 6 & 1 & 0.67 & 0.17 & 0 & 0 & 0.17 & 1 \\
6 & 2 & 0 & 0.33 & 0 & 0.5 & 0.17 & 1 \\
6 & 3 & 0.17 & 0.17 & 0.17 & 0.17 & 0.33 & 1 \\
7 & 4 & 0 & 0.29 & 0.14 & 0.29 & 0.29 & 1 \\
7 & 5 & 0.14 & 0 & 0.57 & 0.14 & 0.14 & 1 \\
\hline
\end{tabular}

Fuente: elaboración y cálculos propios con base en datos de INEGI (1994, 1999, 2004, 2005 y 2009). 
Con resultados similares, la dinámica de transición del 2003 al 2008, refleja cambios en el nivel de posicionamiento de la productividad, aunque las tendencias se mantienen en cierto modo. Por ejemplo, el primer rango presenta una mayor probabilidad de quedarse en el mismo nivel que transitar hacia otro más productivo. De manera contraria, el intervalo de mayor productividad presenta una menor probabilidad de que las entidades mantengan su posición, lo cual se traduce en que es más probable que disminuyan su nivel productivo a mantenerlo con altos indicadores.

Para este periodo las entidades que se ubicaban en el primer intervalo, las cuales se mantuvieron, fueron Baja California Sur, Campeche, Guerrero y San Luis Potosí; las que mejoraron fueron Nuevo León y Zacatecas. Para el segundo rango, Baja California y Durango se mantuvieron, mientras que Guanajuato, Morelos y Sinaloa mejoraron su posición.

En cuanto al tercer rango, únicamente la entidad que se mantuvo en su sitio fue Tabasco, mientras que Coahuila, Distrito Federal y México mejoraron su posición; Chihuahua y Veracruz la empeoraron.

Para el cuarto intervalo se tiene que Hidalgo y Querétaro se mantuvieron, Aguascalientes y Sonora mejoraron mientras que Michoacán, Querétaro y Oaxaca empeoraron. En lo que respecta al quinto rango, se tiene que sólo Puebla mantuvo su posición; en cambio Colima, Chiapas, Jalisco, Nayarit, Tamaulipas y Yucatán la empeoraron.

La tabla 4 muestra las probabilidades de transición de todo el periodo de análisis, es decir de 1993 al 2008.

\section{Tabla 4}

\section{Matriz de Transición 1993-1998}

\begin{tabular}{|c|c|c|c|c|c|c|c|}
\hline $\begin{array}{l}\text { Núm. } \\
\text { Entidades }\end{array}$ & Intervalos & 1 & 2 & 3 & 4 & 5 & Suma \\
\hline 6 & 1 & 0.5 & 0.17 & 0 & 0.17 & 0.17 & 1 \\
\hline 6 & 2 & 0.17 & 0.5 & 0 & 0.17 & 0.17 & 1 \\
\hline 6 & 3 & 0.17 & 0.17 & 0.33 & 0.17 & 0.17 & 1 \\
\hline 7 & 4 & 0.14 & 0.14 & 0.14 & 0.29 & 0.29 & 1 \\
\hline 7 & 5 & 0 & 0 & 0.43 & 0.29 & 0.29 & 1 \\
\hline
\end{tabular}

Fuente: elaboración y cálculos propios con base en datos de INEGI (1994, 1999, 2004, 2005 y 2009).

La transición más drástica o del periodo más amplio es la correspondiente a la tabla 4, pues comprende casi 15 ańos desde la firma del TLCAN hasta el último censo registrado; las probabilidades de cambio entre los intervalos varían entre cada rango, aún así en la mayoría de los casos existe mayor probabilidad de permanecer en el rango inicial que 
moverse a uno de mayor rendimiento en productividad, a excepción del último intervalo, el cual muestra que hay mayor probabilidad de transición a un intervalo intermedio que de mantenerse en el de mayor productividad. Esto último se interpreta como que es más probable que las entidades disminuyan su productividad en lugar de mantenerse con un alto nivel de ese indicador.

Por otra parte, como lo indica la metodología y las propiedades de las cadenas de Markov, con este instrumento se pueden obtener las probabilidades de transición para cualquier estado en un tiempo futuro; considerando la última matriz 2003-2008 se puede hacer una proyección para el año $2013^{8}$ simplemente elevando al cuadrado los valores de dicha matriz, la cual queda como a continuación se muestra:

\section{Tabla 5}

Matriz de segundo estado de transición 2003-2008

\begin{tabular}{ccrcrrr}
\hline $\begin{array}{c}\text { Núm. } \\
\text { Entidades }\end{array}$ & Intervalos & \multicolumn{1}{c}{1} & 2 & 3 & 4 & 5 \\
\hline 6 & 1 & 0.44 & 0.03 & 0 & 0 & 0.03 \\
6 & 2 & 0 & 0.11 & 0 & 0.25 & 0.03 \\
6 & 3 & 0.03 & 0.03 & 0.03 & 0.03 & 0.11 \\
7 & 4 & 0 & 0.08 & 0.02 & 0.08 & 0.08 \\
7 & 5 & 0.02 & 0 & 0.33 & 0.02 & 0.02 \\
\hline
\end{tabular}

Fuente: elaboración y cálculos propios con base en datos de INEGI (1994, 1999, 2004, 2005 y 2009).

La tabla 5 nos da una proyección de lo que sería la dinámica para el año 2013, la cual tiene características diferentes a las anteriores pero en esencia la lectura o interpretación es la misma. En este caso, las entidades que se ubican en el primer intervalo tienen $44 \%$ de posibilidades de permanecer en el mismo nivel de productividad y $3 \%$ de trasladarse al segundo y al quinto rango de productividad.

Una situación parecida ocurre en el resto de los intervalos, donde la mayor probabilidad está en permanecer en el mismo rango; esto con excepción del último intervalo, en el que es más probable transitar a un intervalo de menor rendimiento productivo que mantenerse dentro del mismo.

Ahora bien, la evolución de la productividad se describe de acuerdo a la siguiente expresión:

\footnotetext{
${ }^{8}$ El desarrollo de este documento se llevó a cabo antes del último censo correspondiente a 2013, es por ello que se plantea una proyección para 2014 y no se utiliza el dato como tal.
} 


$$
\pi_{t+1}=\pi_{t} P
$$

Y aplicando esta igualdad a nuestra propuesta, obtenemos el vector invariante o ergódico, necesario para evaluar la convergencia productiva regional:

$$
\begin{gathered}
\pi_{t+1}=\pi_{t} P \\
\pi_{t+1}=\pi * \\
\pi_{t+1}= \\
\pi_{1}=\left[\begin{array}{lllll}
0.19 & 0.19 & 0.19 & 0.22 & 0.22
\end{array}\right] *\left[\begin{array}{cccccc}
0.50 & 0.17 & 0.0 & 0.17 & 0.17 \\
0.17 & 0.50 & 0.0 & 0.17 & 0.17 \\
0.17 & 0.17 & 0.17 & 0.33 & 0.17 \\
0.14 & 0.14 & 0.14 & 0.29 & 0.29
\end{array}\right] \\
\pi^{*}=\left[\begin{array}{lllll}
0.19 & 0.19 & 0.19 & 0.19 & 0.20
\end{array}\right]
\end{gathered}
$$

El ejemplo numérico anterior, únicamente obtiene el vector ergódico para la matriz de transición de 2003 a 2008, pero dado que éste se puede obtener para cualquier año, los vectores ergódicos correspondientes de las matrices de transición calculadas previamente son los siguientes:

$$
\begin{gathered}
\pi_{93-98}=\left[\begin{array}{lllll}
0.19 & 0.19 & 0.19 & 0.22 & 0.22
\end{array}\right] \\
\pi_{98-03}=\left[\begin{array}{lllll}
0.16 & 0.22 & 0.19 & 0.22 & 0.22
\end{array}\right] \\
\pi_{98-03}=\left[\begin{array}{lllll}
0.1904 & 0.19 & 0.19 & 0.2215 & 0.223
\end{array}\right]
\end{gathered}
$$

En este contexto, Quilis (1997) afirma que el vector ergódico debe tener una distribución unimodal para no rechazar la hipótesis de la convergencia. Si es multimodal se dice que no hay convergencia. En particular, el caso bimodal indica la presencia de atractores locales en regiones, mismos que definen los clubes de convergencia formados por regiones ricas y pobres o polarizadas como lo menciona Quah (1996), pero que este resultado no es sinónimo de convergencia.

El caso concreto de esta aplicación de cadenas de Markov, para la productividad del trabajo en las entidades de México, muestra resultados no muy distintos de aquellas primeras aproximaciones que habíamos observado en los mapas. Para el primer caso de acuerdo al vector er- 
gódico (1993-1998) se tiene una distribución bimodal, lo cual indica un cierto grado de polarización al interior del país en cuanto a su productividad. Sin embargo, para el caso de la transición 2003-2008, el vector ergódico indica una distribución unimodal, característica que nos puede decir en cierta medida que al menos en productividad hubo convergencia, situación reflejada en el mapa 4 cuando disminuyen los colores contrastantes y aumentan los tonos intermedios.

Resumiendo, la dinámica productiva en todas las fases de transición desde 1993 a 2008, tiene una distribución ergódica bimodal, ${ }^{9}$ por lo tanto se rechaza la hipótesis de convergencia en México desde 1993 a 2008. Al mismo tiempo hay evidencia empírica de que se ha presentado un proceso de polarización interna entre las entidades federativas que, en palabras de Quilis (1997), indica la asociación de regiones pobres y ricas, que en su interacción se trata de regiones productivamente distantes o polarizadas.

\section{Dinámica distributiva de la industria maquiladora de exportación}

La industria maquiladora en México es una parte muy relevante de la manufactura y del empleo formal, por ello que es necesario distinguir su comportamiento, dinámica y distribución. Para analizar esta industria es necesario utilizar una base de datos diferente a la referida previamente, en este caso se trata de la Encuesta Maquiladora de Exportación (IME).

Para la maquiladora no se cuenta con una base de datos homogénea de participación de las entidades ${ }^{10}$ sin embargo fue posible realizar el mismo ejercicio de dinámica de distribución, lo importante es identificar si la maquiladora presenta el mismo comportamiento que la industria en general.

Dado que la IME aporta datos mensuales, para evaluar de 1996 a 2006, se procedió a obtener un promedio anual para tres cortes en el tiempo; las cadenas de Markov se realizaron de 1996-2000, 2000-2006 y 19962006. ${ }^{11}$ El vector de estado inicial y las matrices de transición se muestran a continuación.

\footnotetext{
${ }^{9}$ La distribución bimodal se atribuye a que, al considerar los tres vectores ergódicos, seis observaciones dieron un coeficiente de 0.19 y otras seis uno de 0.22 , una de 0.18 y uno más de 0.16 .

${ }^{10}$ Las entidades que cuentan con datos para la maquiladora son Aguascalientes, Baja California, Baja California Sur, Chihuahua, Coahuila, Distrito Federal, Durango, Guanajuato, Jalisco, México, Nuevo León, Puebla, San Luis Potosí, Sinaloa, Sonora, Tamaulipas, Yucatán y Zacatecas.

${ }^{11}$ Los intervalos temporales que se consideran en el ejemplo maquilador se tomaron al obtener únicamente el valor intermedio del periodo 1996-2006, es por ello que se hace el corte temporal en el 2000 .
} 


$$
\pi_{2}=\left[\begin{array}{llll}
0.28 & 0.28 & 0.28 & 0.17
\end{array}\right]
$$

El vector de estado inicial indica que para las entidades maquiladoras hay $28 \%$ de probabilidades de ubicarse en el rango de menor nivel productivo, la misma proporción para los rangos dos y tres, sin embargo para el cuatro hay una probabilidad de $17 \%$, es decir, hay mayores posibilidades para las regiones maquiladoras de localizarse en niveles bajos de productividad. Las matrices de transición para evaluar su dinámica se presentan a continuación.

\section{Tabla 6}

Matriz de Transición de la IME 1996-2000

\begin{tabular}{rrrrr}
\hline & 1 & \multicolumn{1}{c}{2} & \multicolumn{1}{c}{3} & \multicolumn{1}{c}{4} \\
\hline 1 & 0.2 & 0.8 & 0 & 0 \\
2 & 0.4 & 0 & 0.4 & 0.2 \\
3 & 0 & 0.2 & 0.4 & 0.4 \\
4 & 0.33 & 0.00 & 0.33 & 0.33 \\
\hline
\end{tabular}

Fuente: elaboración y cálculos propios con base en datos de INEGI (1994, 1999, 2004, 2005 y 2009).

Debido a que no se cuenta con la totalidad de las entidades para el análisis de la maquiladora, sino únicamente con 18 , la muestra fue dividida en cuatro intervalos; la interpretación de la tabla 6 se lee de manera similar a lo antes discutido, es decir, para las entidades maquiladoras que se encuentran en el rango de menor productividad (intervalo 1) existe $20 \%$ de posibilidades de permanecer en el intervalo menos productivo y $80 \%$ de transitar al segundo nivel.

Para las entidades localizadas en el segundo intervalo hay $40 \%$ de probabilidades de permanecer en ese rango, otro $40 \%$ de ascender al rango tres y $20 \%$ de subir hasta el intervalo cuatro que es el de mayor productividad.

La tabla 6, en su mayoría, presenta la misma tendencia y lectura del análisis de la industria manufacturera total, donde la mayor probabilidad de los intervalos se concentró en mantenerse en el mismo nivel productivo, esto es que la diagonal principal de la matriz arroja las proporciones de participación más altas; sin embargo para el caso maquilador 19962000 , en el segundo intervalo ninguna entidad se mantiene en este nivel, lo cual refleja cierta mejoría, pues hubo más probabilidades de aumentar el rendimiento en productividad.

Para el caso del periodo 2000-2006, la matriz de transición se muestra a continuación. 
Tabla 7

Matriz de Transición de la IME 2000-2006

\begin{tabular}{rrrrr}
\hline & 1 & 2 & 3 & 4 \\
\hline 1 & 0.6 & 0.2 & 0 & 0.2 \\
2 & 0.2 & 0.4 & 0.4 & 0 \\
3 & 0.2 & 0.4 & 0.4 & 0 \\
4 & 0.33 & 0.00 & 0.33 & 0.33 \\
\hline
\end{tabular}

Fuente: elaboración y cálculos propios con base en datos de INEGI (1994, 1999, 2004, 2005 y 2009).

La interpretación de la tabla 7 nos dice que para las entidades maquiladoras hay $60 \%$ de probabilidades de permanecer en el primer intervalo (proporción bastante alta comparada con el resto), 20\% de transitar hacia el segundo intervalo y $20 \%$ hacia el intervalo cuatro; así sucesivamente, el resto de los rangos en productividad donde nuevamente la diagonal principal reafirma que hay más probabilidades de permanecer en el nivel de productividad inicial.

Ahora bien, para analizar el proceso dinámico de las entidades maquiladoras, y evaluar si tienden hacia una convergencia o divergencia, es necesario multiplicar el vector de estado inicial $(\pi 2)$ por las matrices de transición previas, para ambos casos los vectores ergódicos se muestran a continuación:

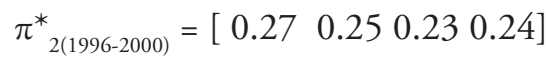

$$
\begin{aligned}
& \pi_{2(2000-2006)}^{*}=\left[\begin{array}{llll}
0.25 & 0.27 & 0.27 & 0.24
\end{array}\right]
\end{aligned}
$$

Con lo anterior se percibe que la distribución de la productividad del trabajo en la industria maquiladora sigue una dinámica divergente, hasta cierto punto se esperaba este resultado, puesto que la manufactura total tampoco tendió a la convergencia, sólo que en el caso manufacturero la dinámica fue polarizada, mientras que la maquiladora fue totalmente divergente, es decir que no hay clubes de convergencia o polos, este resultado se corrobora al no encontrar una distribución unimodal en los vectores ergódicos previos.

\section{Conclusiones}

La mayoría de los estudios que han aportado evidencia empírica al respecto del proceso de la convergencia regional en México han basado primordialmente sus análisis en técnicas paramétricas, utilizando como 
variable central al ingreso per cápita por entidad. Con este tipo de metodología, y en sus diversas variantes, se ha confirmado que en el caso de las regiones de México, más que un proceso de convergencia lo que se ha presentado es un proceso de divergencia y polarización con algunos rasgos de la denominada "convergencia de clubes", la cual se traduce en que las regiones de mayor ingreso convergen con regiones similares y las de menor ingreso con las más pobres.

Por otra parte, la productividad del trabajo es una variable que ha sido considerada como un elemento detonante del proceso de convergencia/ divergencia regional, pero a pesar de tales afirmaciones, el análisis de la convergencia que considera este indicador aún es muy escaso.

En este documento se analizó la distribución y la dinámica de la productividad del trabajo en la industria manufacturera y maquiladora en México por entidad federativa, con lo que se pudieron obtener importantes conclusiones que no distan de la realidad ya observada.

En primera instancia, la heterogeneidad en la distribución de la manufactura y maquiladora, y por ende la productividad del trabajo para este sector, hace que las entidades se perfilen hacia la confirmación de un proceso de divergencia, dado que esta industria presenta una mayor concentración en las entidades del centro y norte del país. La dinámica de transición o la evolución de la productividad en los diferentes ańos observados dan pie a algunos resultados contrastantes entre cada periodo.

En particular, en 1998, la distribución productiva marcaba una clara divergencia entre cada entidad, pero ya en el último dato censal se observan tenues indicios de convergencia productiva. Desde luego que esto ocurre cuando se considera únicamente la distribución espacial de dicha variable.

En cuanto a la metodología y aplicación técnica también es importante reconocer la propuesta alternativa para evaluar la convergencia/ divergencia con el uso de las técnicas de análisis no paramétricas, como son las cadenas de Markov, relativamente escasas en México, y mediante las cuales se pudieron contrastar los resultados obtenidos con algunas otras investigaciones, al respecto se encontraron algunas similitudes en los resultados.

Utilizando la técnica no paramétrica fue posible analizar la dinámica de transición de la productividad de un estado inicial a uno final; concretamente, en la mayoría de los casos, hubo cambios en la dinámica distributiva.

Sin embargo, se reconoce que hay una tendencia a permanecer en las condiciones iniciales de la productividad tanto para el caso manufacturero como para el maquilador, esto significa, fundamentalmente, que 
es más probable mantenerse en un rango de baja productividad que trasladarse hacia un rango más elevado.

En cambio, es más probable transitar de un rango de alta productividad hacia uno de menor jerarquía que mantenerse en un nivel alto de rendimiento. Es decir, que la transición es más probable que se incline hacia la baja productividad que hacia la alta. Estas percepciones, en cuanto a tendencias y probabilidades de transición, sólo pueden ser evaluadas con el uso de este tipo de metodologías, reconociendo aquí otra de las bondades de las cadenas de Markov que justifican en parte el uso de este método alternativo.

Con todo lo anterior, y al considerar el análisis de distribución y dinámica de transición de la productividad entre las entidades a raíz de la firma del TLCAN, se ha podido evaluar el proceso de convergencia entre las regiones en México; los resultados muestran una clara tendencia hacia la divergencia en el caso de la maquiladora y, por el contrario, un comportamiento polarizado para la manufactura no maquiladora. No obstante, cabe señalar que para periodos más cortos se ha logrado observar cierta convergencia, precisamente entre 2003 y 2008, esto únicamente para el caso no maquilador.

El documento ha contribuido a reforzar la afirmación de que en México, después de la firma del TLCAN, no sólo no se ha logrado un proceso de convergencia productiva, sino que en su lugar se ha consolidado un proceso de divergencia entre regiones, polarizando cada vez más el centronorte con el sur-sureste, una situación que claramente no es favorable para mejorar las condiciones de desarrollo y crecimiento económico del país, estos resultados confirman los hallazgos de convergencia regional basados en métodos paramétricos. 
Anexo

Productividad del trabajo manufacturero por entidad y ańo

\begin{tabular}{lllll}
\hline \multicolumn{1}{c}{ Entidad } & 1993 & 1998 & 2003 & 2008 \\
\hline Aguascalientes & 1.85 & 1.80 & 2.92 & 3.60 \\
Baja California & 1.57 & 1.40 & 1.81 & 2.17 \\
Baja California Sur & 0.87 & 1.10 & 1.18 & 1.25 \\
Campeche & 0.54 & 0.67 & 0.79 & 0.74 \\
Chiapas & 2.19 & 2.37 & 2.70 & 4.68 \\
Chihuahua & 1.14 & 2.63 & 3.32 & 2.55 \\
Coahuila & 0.99 & 0.97 & 4.67 & 3.54 \\
Colima & 1.37 & 1.23 & 2.34 & 2.14 \\
Distrito Federal & 2.64 & 1.75 & 2.01 & 3.22 \\
Durango & 1.11 & 1.33 & 1.41 & 2.25 \\
Guanajuato & 1.57 & 1.92 & 2.84 & 2.81 \\
Guerrero & 0.84 & 0.67 & 0.87 & 0.39 \\
Hidalgo & 2.20 & 2.14 & 3.75 & 3.39 \\
Jalisco & 2.93 & 2.07 & 2.33 & 2.70 \\
México & 2.97 & 2.70 & 3.23 & 3.77 \\
Michoacán & 1.51 & 1.49 & 1.46 & 2.40 \\
Morelos & 4.50 & 3.28 & 4.94 & 3.33 \\
Nayarit & 1.54 & 1.97 & 1.32 & 1.35 \\
Nuevo León & 2.51 & 2.24 & 3.30 & 4.06 \\
Oaxaca & 4.83 & 1.87 & 4.97 & 1.92 \\
Puebla & 1.35 & 1.41 & 2.95 & 2.79 \\
Querétaro & 2.55 & 1.68 & 3.00 & 4.08 \\
Quintana Roo & 1.30 & 1.12 & 1.25 & 2.05 \\
San Luis Potosí & 2.24 & 2.90 & 2.74 & 3.54 \\
Sinaloa & 2.23 & 1.58 & 1.52 & 1.90 \\
Sonora & 2.20 & 2.23 & 2.16 & 3.32 \\
Tabasco & 2.43 & 5.25 & 8.93 & 8.30 \\
Tamaulipas & 1.79 & 1.58 & 2.05 & 2.49 \\
Tlaxcala & 1.81 & 1.83 & 2.36 & 2.52 \\
Veracruz & 3.41 & 2.69 & 3.62 & 1.25 \\
Yucatán & 1.13 & 1.00 & 1.16 & 7.83 \\
Zacatecas & 0.85 & 1.64 & 2.53 & 2.80 \\
\hline
\end{tabular}

Productividad $=$ Valor Agregado Censal/ Personal ocupado

El Valor Agregado Censal fue deflactado con el deflactor implícito del piB para las actividades manufactureras por entidad para 1993, 1998 y 2003 a precios del 2008.

Fuente: elaboración propia con base en datos del INEGI (1994, 1999, 2004 y 2009). 


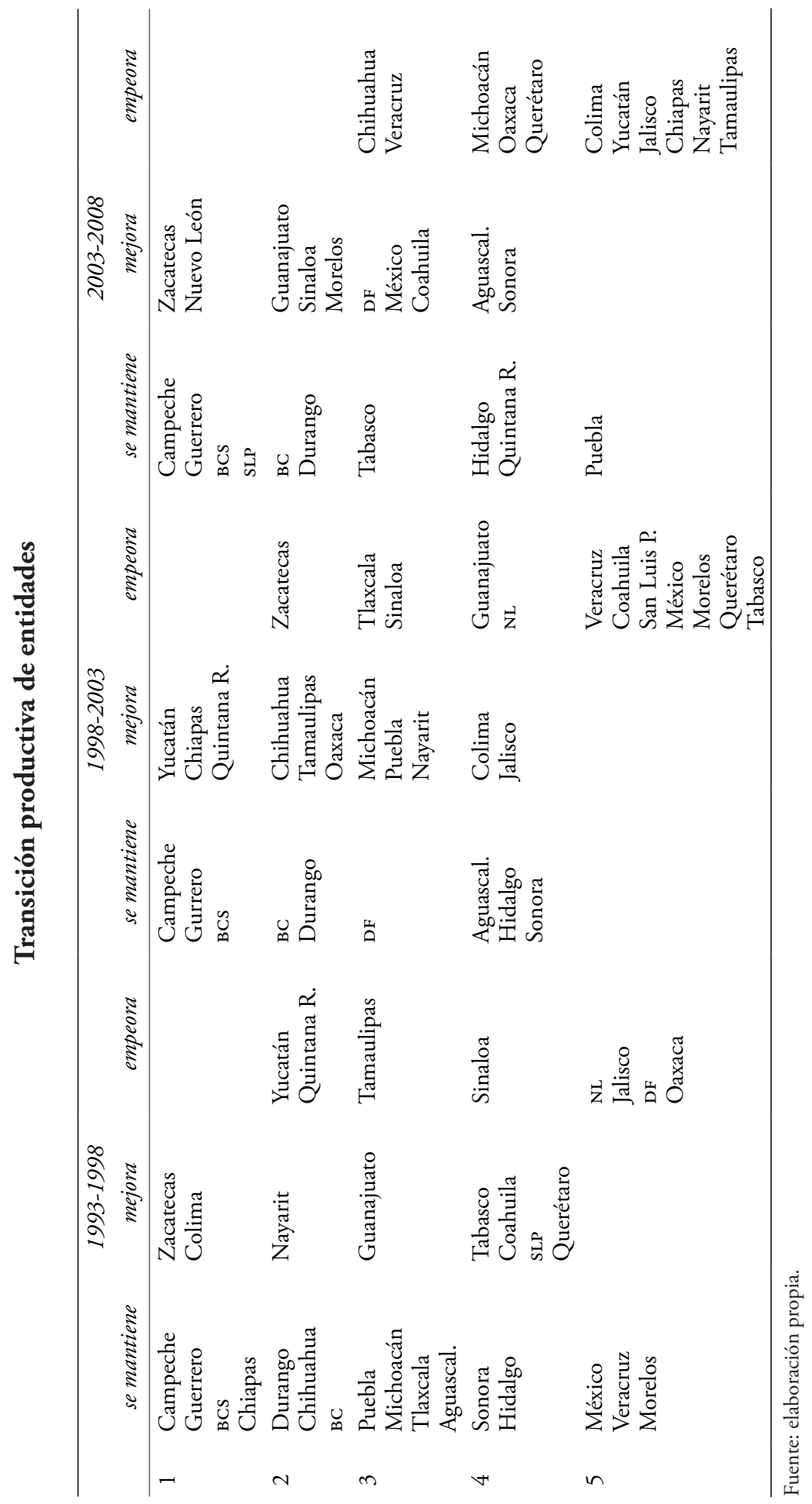




\section{Fuentes consultadas}

Alañon, Ángel Pardo (1999), "El uso práctico de las técnicas de econometría espacial: la productividad del trabajo industrial”, documento de trabajo núm. 9908, Facultad de Ciencias Económicas y Empresariales, Universidad Complutense de Madrid, Madrid.

Aroca, Patricio, William Maloney y Mariano Bosch (2003), "Is NAFTA polarizing Mexico? Or el sur también existe? Spatial dimensions of Mexico's post-liberalization growth", Social Science Research Network, Washington, <doi:10.2139/ssrn.402440>.

Arriaga-Navarrete, Rosalinda, Elitania Leyva Rayón, José Luis Estrada López (2005), "Perfil y estructura industria de Guanajuato y Querétaro: un análisis de la producción, el empleo y los salarios", Análisis Económico, XX (044), Universidad Autónoma Metropolitana, México, pp. 135-189.

Arrufat, José Luis, A. J. Figueras, J. V. Blanco y M. D. de la Mata (2005), "Análisis de la movilidad regional en Argentina: un enfoque basado en cadenas de Markov", ponencia presentada en XL Anales de la Reunión Anual de la Asociación Argentina de Economía Política, noviembre de 2005, La Plata.

Barro, Robert J., Xavier Sala-i-Martin, Oliver Jean Blanchard, Roberto E. Hall (1991), "Convergence across states and regions", Brookings Papers on Economic Activity, 191 (1), The Brooking Institution, Washington, pp. 107-182.

Bedoya Juan Carlos y Mauricio Barrera (2006), "Convergencia de las cadenas de Markov", Scientia et technica, XII (32), Universidad Tecnológica de Pereira, Pereira, pp.73-78.

Cáceres-Apolinario, Rosa María, Santiago Rodríguez Feijoó, Alejandro Rodríguez Caro, Octavio Maroto Santana (2009), "Análisis de convergencia regional de los países con economías emergentes: un estudio con cadenas de Markov", ponencia presentada en la XXXV Reunión de estudios regionales, Asociación Española de Ciencia Regional, 26 y 27 de noviembre, Valencia.

Calderón Cuauhtémoc y Anna Tykhonenko (2006), "La liberalización económica y la convergencia regional en México", Comercio ex- 
terior, 56 (5), Banco Nacional de Comercio Exterior, México, pp. 374-381.

Dall'erba, Sandy (2005), "Productivity convergence and spatial dependence among spanish regions", Journal of Geographical Systems, 7 (2), Springer-Verlag, Berlín, pp. 207-227, <doi:10.1007/s10109005-0157-4>.

Dávila-Flores, Alejandro (2005), "México: concentración y localización del empleo manufacturero, 1980-1998”, Economía Mexicana, XII (2), Centro de Investigación y Docencia Económicas, México, pp. 209-254.

De León-Arias, Adrián (2008), "Cambio regional del empleo y productividad manufacturera en México, el caso de la frontera norte y las grandes ciudades: 1970-2004”, Frontera Norte, 20 (40), El Colegio de la Frontera Norte, Tijuana, pp. 70-103.

Díaz-Bautista, Alejandro (2008) "Divergencia regional en los niveles de productividad sectorial del trabajo y total factorial", Comercio exterior, 58 (3), Banco Nacional de Comercio Exterior, México, pp. 187-197.

Durlauf, Steven y Danny Quah (1998), "The new empirics of economic growth”, working paper 6422, National Bureau of Economic Research, Cambridge, <doi:10.3386/w6422>.

Esquivel, Gerardo y Miguel Messmacher (2002), "Sources of regional (non) convergence in Mexico", World Bank, Washington.

Fragoso-Pastrana, Edna (2003), "Apertura comercial y productividad en la industria manufacturera mexicana", Economía Mexicana, XII (001), Centro de Investigación y Docencia Económicas, México, pp. 5-38.

García-Verdú, Rodrigo (2005), "Income, mortality and literacy distribution dynamics across states in Mexico: 1994-2000", Cuadernos de economia. Latin American Journal of Economics, 42 (125), Pontificia Universidad Católica de Chile, Santiago de Chile, pp. 165-192.

Gutiérrez-Flores, Luis, Alba Veronica Méndez-Delgado y José Refugio Reyes-Valdés (2011), "La movilidad y la distribución del ingreso 
en los municipios de Coahuila”, Región y Sociedad, XXIII (52), El Colegio de Sonora, Hermosillo, pp. 131-171.

INEgi (Instituto Nacional de Estadística y Geografía) (2009), Censos Económicos 2009, INEGI, México.

INEgi (Instituto Nacional de Estadística y Geografía) (2005), Marco Geoestadístico Municipal Versión 1.0, INEGI, México.

INEGI (Instituto Nacional de Estadística y Geografía) (2004), Censos Económicos 2004, INEGI, México.

INEgi (Instituto Nacional de Estadística y Geografía) (1999), Censos Económicos 1999, INEGi, México.

INEGI (Instituto Nacional de Estadística y Geografía) (1994), Censos Económicos 1994, INEgI, México.

Ocegueda-Hernández, Juan Manuel (2007), "Apertura comercial y crecimiento económico en las regiones de México", Investigación económica, LXVI (262), Universidad Nacional Autónoma de México, México, pp. 89-137.

Ponce de León, Andrés (2012), “Convergencia regional: México después del TLCAN”, documento de trabajo núm. 423, Fundación Rafael Preciado Hernández-CEDispan, México.

Quah, Danny (1993), "Empirical cross-section dynamics in economic growth", European Economic Review, 37, Elsevier, North Holland, pp. 426-434.

Quah, Danny (1996), “Twin picks: growth and convergence in models of distribution dynamics", The economic journal, 106 (437), Royal Economic Society, Oxford, pp.1045-1055.

Quilis, Enrique (1997), “Convergencia de la productividad en España, un análisis dinámico de su distribución regional”, manuscrito del Instituto Nacional de Estadística, Madrid, <http://www.ine.es/ daco/daco42/daco4214/cbtc13.pdf>, 12 de octubre de 2012.

Rodriguez-Arana, Alejandro (2009), "Apertura comercial, balanza comercial e inversión extranjera directa en México 1980-2006”, Inves- 
tigación económica, LXVIII (269), Universidad Nacional Autónoma de México, México, pp. 73-111.

Sobrino, Jaime (1999) "El contexto espacial de las actividades económicas", documento de investigación, núm. 30, El Colegio Mexiquense, A.C., Zinacantepec.

Trejo-Nieto, Alejandra Berenice (2010), "The aftermath of openness and integration in the mexican manufacturing", Jahrbuch für Regionalwissenschaft, 30 (1), Springer Verlag, Berlín, pp. 23-44, <doi:10.1007/s10037-009-0041-7>.

Valdivia-López Marcos (2007), "Heterogeneidad espacial, convergencia y crecimiento regional en México", ponencia presentada en el XVII Coloquio de Economía Matemática y Econometría, 21-25 de mayo, Universidad de Quintana Roo, Quintana Roo.

Velázquez, Maritza (2010), "Productividad del trabajo y dinámica salarial en la industria manufacturera para las entidades federativas en México, 1994-2008”, tesis de maestría, Universidad Autónoma de Coahuila. Saltillo.

Recibido: 23 de enero de 2013. Reenviado: 16 de julio de 2014. Aceptado: 13 de junio de 2015.

Luis Gutiérrez-Flores. Doctor en economía por la Universidad Autónoma de Baja California. Actualmente es director y profesor del Centro de Investigaciones Socioeconómicas de la Universidad Autónoma de Coahuila. Es miembro del Sistema Nacional de Investigadores en el área de Ciencias Sociales. Sus principales intereses de investigación son los temas relacionados con la distribución del ingreso, la desigualdad, la movilidad y la pobreza. Asimismo, ha escrito sobre la aplicación del análisis económico regional, el crecimiento económico y los efectos de la innovación sobre la productividad regional. Algunas de su publicaciones son: en coautoría, "A standarized coefficients model to analyse the regional patents activity: evidence from the mexican states", Journal of the Knowledge Economy, vol. 6, Springer, Berlín, pp. $72-89$ (2015); "La importancia de la infraestructura física en el crecimiento económico de los municipios de la frontera norte", Estudios fronterizos, 13 (25), Universidad Autónoma de Baja California, Baja California, pp. 57-88 (2012). 
Maritza Areli Velázquez-Villalpando. Doctora en Economía Regional por la Universidad Autónoma de Coahuila. Sus principales intereses de investigación se enfocan a analizar la productividad del trabajo en el sector manufacturero, la distribución regional de productividad y salarios, la eficiencia técnica. También ha desarrollado otros trabajos de investigación referentes a la planeación regional y municipal en el Estado de México. Docente en licenciatura y maestría en el área de estadística y políticas públicas.

Gustavo Félix-Verduzco. Doctor en Economía por la Universidad Autónoma de Barcelona. Profesor-Investigador del Centro de Investigaciones Socioeconómicas de la Universidad Autónoma de Coahuila. Miembro del Sistema Nacional de Investigadores. Su investigación se centra en la econometría aplicada con énfasis en el análisis regional de la industrialización, pobreza y economía laboral, es coordinador del libro Dimensiones socioeconómicas de la pobreza en México, Plaza y Valdés-Universidad Autónoma de Coahuila, México (2012). 